\title{
Entanglement Swapping with Photons Generated on Demand by a Quantum Dot
}

\author{
F. Basso Basset $\odot,{ }^{1, *}$ M. B. Rota, ${ }^{1, *}$ C. Schimpf, ${ }^{2, *}$ D. Tedeschi, ${ }^{1, *}$ K. D. Zeuner, ${ }^{3}$ S. F. Covre da Silva, ${ }^{2}$ \\ M. Reindl, ${ }^{2}$ V. Zwiller, ${ }^{3}$ K. D. Jöns, ${ }^{3}$ A. Rastelli, ${ }^{2}$ and R. Trotta ${ }^{1, \dagger}$ \\ ${ }^{1}$ Department of Physics, Sapienza University of Rome, 00185 Rome, Italy \\ ${ }^{2}$ Institute of Semiconductor and Solid State Physics, Johannes Kepler University, 4040 Linz, Austria \\ ${ }^{3}$ Department of Applied Physics, Royal Institute of Technology, 10691 Stockholm, Sweden
}

(Received 19 February 2019; published 14 October 2019)

\begin{abstract}
Photonic entanglement swapping, the procedure of entangling photons without any direct interaction, is a fundamental test of quantum mechanics and an essential resource to the realization of quantum networks. Probabilistic sources of nonclassical light were used for seminal demonstration of entanglement swapping, but applications in quantum technologies demand push-button operation requiring single quantum emitters. This, however, turned out to be an extraordinary challenge due to the stringent prerequisites on the efficiency and purity of the generation of entangled states. Here we show a proof-of-concept demonstration of all-photonic entanglement swapping with pairs of polarization-entangled photons generated on demand by a GaAs quantum dot without spectral and temporal filtering. Moreover, we develop a theoretical model that quantitatively reproduces the experimental data and provides insights on the critical figures of merit for the performance of the swapping operation. Our theoretical analysis also indicates how to improve stateof-the-art entangled-photon sources to meet the requirements needed for implementation of quantum dots in long-distance quantum communication protocols.
\end{abstract}

DOI: 10.1103/PhysRevLett.123.160501

Entanglement swapping has been observed in a few different systems, from the original all-photonic scheme that employs a spontaneous parametric down-conversion (SPDC) source [1] to hybrid protocols in which the interference of two photons is used to entangle spins [2] or atoms [3] at a distance. The swapping procedure between pairs of photons is especially relevant to the development of future quantum networks, because it provides a way to overcome the absence of optical communication amplifier for photonic qubits due to the no-cloning theorem and to create entanglement over distances beyond the reach of direct transmission $[4,5]$.

Developing quantum light sources able to operate on demand is an important step towards this goal. Despite the impressive technological achievements up to date [6], SPDC sources are limited by the probabilistic nature of the photon generation process [7]. Quantum emitters, such as atoms, nitrogen vacancies in diamonds, and semiconductor quantum dots (QDs), overcome this hurdle and hold strong promise for deterministic operation. Among these, the latter are receiving attention after recent reports of QD-based single-photon sources overtaking SPDC in terms of brightness, single

Published by the American Physical Society under the terms of the Creative Commons Attribution 4.0 International license. Further distribution of this work must maintain attribution to the author(s) and the published article's title, journal citation, and DOI. photon purity, and indistinguishability [8,9]. In addition, they are closing the performance gap concerning the generation of polarization-entangled photons as well [10-14], leading to the recent demonstration of three-photon quantum teleportation [15], even under deterministic photon generation [16].

However, to the best of our knowledge, there is no report on the use of QDs - and more generally of any solid-statebased quantum emitter-for entanglement swapping using photon pairs. This absence of experimental results is likely related to the outstanding challenges set by the implementation of four-photon swapping protocols involving high quality entangled-photon pairs. Below, we detail the specific challenges and explain how they can be successfully overcome.

Using a single QD we design an entanglement swapping experiment which follows the seminal work of Pan et al. [1], as illustrated in the inset of Fig. 1. Photon pairs in the maximally entangled state $\left|\Phi^{+}\right\rangle=1 / \sqrt{2}(|H H\rangle+|V V\rangle)$ are deterministically generated from a QD with exciton fine structure splitting (FSS) well below the radiative-limited excitonic linewidth [17] by exciting the radiative biexcitonexciton $(X X-X)$ cascade via a two-photon resonant scheme [18-20]. Two $X X-X$ entangled-pairs $\left(\left|\Phi_{E}^{+}\right\rangle\right.$and $\left|\Phi_{L}^{+}\right\rangle$, linked to the early and late generation pulse) are independently triggered by two subsequent laser pulses, and the photons emitted by the $X$ transition $\left(X_{E}\right.$ and $\left.X_{L}\right)$ are brought to interfere at a beam splitter using a delay line. When these two photons are perfectly indistinguishable, a joint detection at the two output ports of the beam splitter 


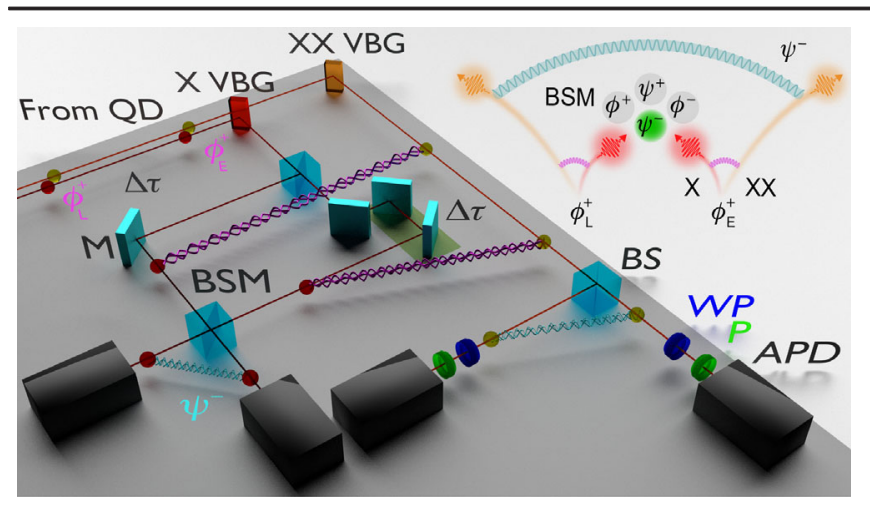

FIG. 1. Schematic illustration of the setup used for the entanglement swapping experiment. $X X$ and $X$ photons are separated by volume Bragg gratings (VBG). $X$ photons are sent into an unbalanced Mach-Zehnder interferometer with an internal delay $\Delta \tau$ matching the time distance between the early- $(E)$ and late- $(L)$ coming entangled pairs. The latter BS of the interferometer performs a partial Bell state measurement (BSM). $X X$ photons are sent to a non-polarizing beam splitter (BS) followed by two sets of wave plate (WP), linear polarizer (P) and singlephoton counting avalanche photodiode (APD) at the two output ports. In the inset, a sketch representing the ideal entanglement swapping process is shown.

corresponds to the detection of the state $\left|\Psi^{-}\right\rangle=$ $1 / \sqrt{2}(|H V\rangle-|V H\rangle)$. This procedure, which implements a partial Bell state measurement (BSM) [21], allows the photons from the other transition $\left(X X_{E}\right.$ and $\left.X X_{L}\right)$ to be projected onto the entangled polarization state $\left|\Psi^{-}\right\rangle$, despite being previously uncorrelated.

This simple picture falls short when a realistic source is considered. Background light, multiphoton emission, finite FSS, and possible decoherence processes can reduce the fidelity to the entangled state $\left|\Phi^{+}\right\rangle[11]$. Likewise, poor photon indistinguishability reduces the purity of the output of the BSM and dramatically affects the swapping operation. In addition, a bright source is essential because entanglement swapping relies on fourfold coincidence events whose rate has a steep fourth-power dependence on the light extraction efficiency (see the derivation and further discussion of the coincidence rates in Sec. SVIII). Meeting all these requirements at the same time has proven to be challenging, precluding any implementation of quantum-emitter-based photonic entanglement swapping up to now.

However, no fundamental limit enforces the figures of merit of current entangled photon sources, and, in this work, we present a solution based on GaAs QDs grown by Al-droplet etching [22-24]. Because of the fast radiative recombination and the weak impact of exciton spin depolarization and dephasing between the two bright exciton states during the intermediate step of the cascade [12,30,31], a fidelity to a maximally entangled Bell state up to $98 \%$ has been reported using these emitters [11]. The use of a resonant two-photon excitation scheme also ensures on demand operation with a preparation fidelity of approximately 90\% [32], excellent suppression of multiphoton emission [33] and good indistinguishability [30]. To improve light collection and enhance the brightness of the source, we integrate the QDs into a monolithic planar cavity composed of two asymmetric distributed Bragg reflectors. This convenient approach maintains the QD optical quality and guarantees a broad bandwidth to deal with the wavelength difference between the $X$ and $X X$ transitions. A light extraction efficiency of $7 \%$ is estimated in the relevant spectral region of emission (see Sec. SVIII for how this figure was calculated).

We selected a QD with optimal trade-off among the relevant figures of merit (details about how the values listed below are measured are in the Secs. SII-SIV $[23,25,26])$. A low FSS $S$ of $0.6(5) \mu \mathrm{eV}$ and an exciton lifetime $\tau_{X}$ of $270(10)$ ps ensure a high degree of $X X-X$ entanglement, as supported by the measured value of Bell state fidelity of 0.88 (2). The Hong-Ou-Mandel (HOM) visibility $V$ of the $X$ line is 0.63(2). Most importantly, a detection rate of approximately $0.5 \mathrm{MHz}$ is achieved on the detectors recording the BSM, which results in a rate of fourfold coincidences of approximately $3 \mathrm{mHz}$, in agreement with our predictions on the throughput of the swapping protocol (whose derivation is described in more depth in Sec. SVIII).

The reported count rates are measured in the setup sketched in Fig. 1. With a repetition rate of $160 \mathrm{MHz}$, a couple of laser pulses separated by a time delay of $1.8 \mathrm{~ns}$ excite the QD and trigger the emission of two pairs of entangled photons, which we label $X X_{E}-X_{E}$ and $X X_{L}-X_{L}$. Volume Bragg gratings are used to separate the photons from the two transitions of the cascade, thus ensuring minimal losses. The photons originating from the exciton to ground state transition, $X_{E}$ and $X_{L}$, are sent to an unbalanced Mach-Zehnder interferometer (with an internal delay also set to $1.8 \mathrm{~ns}$ ) to let them interfere at its second beam splitter. The BSM is thus performed by recording joint detection events between $X_{E}$ and $X_{L}$ within a time window of $0.6 \mathrm{~ns}$. Because of the short radiative lifetime this choice does not postselect the emitted photons by applying a temporal filter on the two-photon interference profile. While this technique is known to increase the HOM visibility [34], it is not suitable for applications as it comes at the cost of decreasing the source brightness. The photons from the biexciton to exciton transition, $X X_{E}$ and $X X_{L}$, are instead sent to a nonpolarizing 50:50 beam splitter and identified at the two output ports based on the arrival time. The polarization optics performs different projective measurements on $X X_{E}$ and $X X_{L}$ so to acquire coincidences in the set of polarization bases required for quantum state tomography. Only the detection events within a temporal range of $100 \mathrm{~ns}$ from a BSM are recorded.

The fourfold coincidences are recorded as a function of the delays between the BSM and the $X X$ detection events on the two tomography channels, as shown in Fig. 2(a) for a 

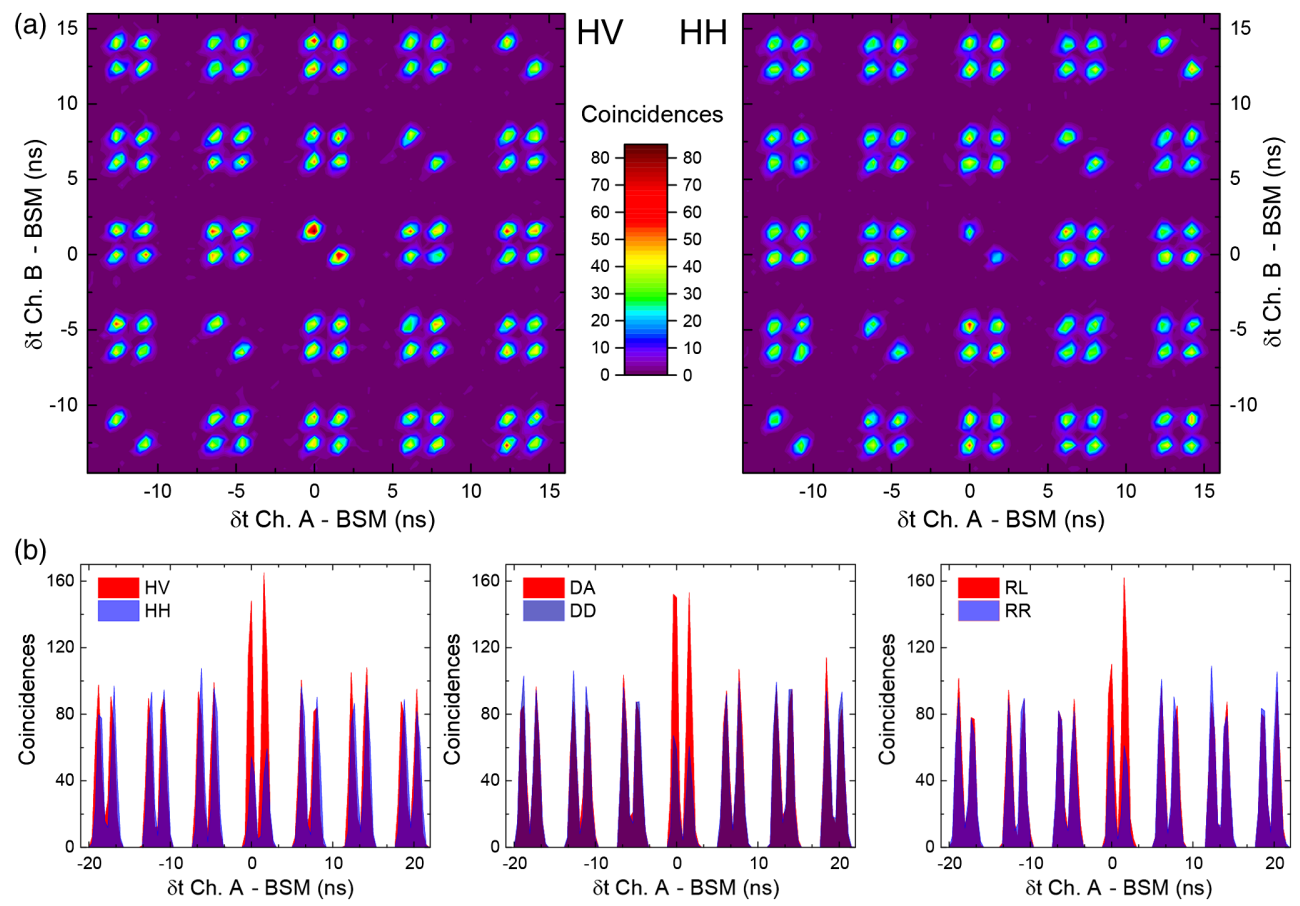

FIG. 2. (a) Fourfold coincidences histograms as a function of the delays between the BSM and the $X X$ detection events on the two tomography channels, recorded for cross- (left) and co-polarized (right) linear polarization. Peaks along the main diagonal would correspond to $X X$ photons excited from the same laser pulse and are therefore absent due to the single photon purity of the QD. The two peaks at the center belong to events synchronized with a BSM and differ only on whether $X X_{E}$ or $X X_{L}$ is detected on channel $A$. Bunching for $\mathrm{HV}$ and antibunching for $H H$ are observed, as expected for the $\left|\Psi^{-}\right\rangle$state. (b) Cross-correlation histograms between $X X$ photons in linear, diagonal, and circular polarization bases. These data are reduced from the fourfold coincidences histograms as presented in panel (a) by binning over the time tags on channel $B$ in the time window included between -1 and $2.8 \mathrm{~ns}$.

pair of co- and cross-polarized $X X$ bases. The comparison between the two peaks near zero delay-which contain the fourfold coincidences of photons coming from two subsequent $X X-X$ cascades-highlights the presence of polarization correlation. To estimate the correlation visibility, the coincidence counts are normalized with respect to the side peaks stemming from $X X$ photons uncorrelated with the BSM, as discussed more in depth in the Sec. SV. In Fig. 2(b) the data are windowed and binned to obtain second-order intensity correlation histograms for the linear, diagonal, and circular bases. The observed bunching and antibunching behaviors clearly show the presence of a swapping process and are consistent with a projection to a state with a dominant $\left|\Psi^{-}\right\rangle$character.

In order to gain complete insight on the result of the swapping operation, we perform the full tomography of the two-photon state and collect $X X_{E^{-}} X X_{L}$ correlations in the 36 possible combinations of linear, diagonal, and circular polarization bases [35]. Note that $X X_{E}$ and $X X_{L}$ are defined by their time of arrival and not by the detector that registers them, therefore permuted pairs of bases are acquired at the same time, and the total number of measurements is reduced to 21 (see Sec. SVI for a list of the performed tomography measurements). The density matrix is reconstructed using a maximum likelihood estimation [36] and is presented in Fig. 3(a).
The raw value of fidelity to the expected Bell state $\left|\Psi^{-}\right\rangle$ is calculated from the density matrix (using the standard formula also reported in Sec. SVI) to be 0.58(4), which indicates a strong correlation between photons that are uncorrelated without the information from the BSM (0.25), surpassing the classical threshold [37] of 0.5 by 2 standard deviations. A consistent evidence of the presence of entanglement is offered by the above-zero raw value of the concurrence, $0.15(8)$. Therefore, our results experimentally demonstrate entanglement swapping between single pairs of entangled photons generated on demand by a quantum emitter. In addition to that, the swapping procedure generates entangled pairs of photons with the same energy and different time bins. These are qualitatively different features with respect to cascaded photons usually observed in QDs.

It is worth emphasizing that the measured level of entanglement between the swapped photons does not consider imperfections stemming from the experimental setup, such as background light and non-ideal beam splitters. Taking these imperfections into account the fidelity would be expected to increase to 0.64(4) and the concurrence to $0.28(8)$, see below.

While our result offers a valid proof of concept, higher levels of entanglement will be needed for real-life quantum communication, which demands a degree of entanglement 
of the swapped photons large enough to violate Bell's inequality (since our outcome of the swapping operation closely resembles a Werner state, a concurrence approximately larger than 0.58 would be needed [38]), and to implement error-correction protocols for secure QKD (fidelity larger than 0.8 [39]). As the temporal [34] and spectral $[40,41]$ filtering techniques usually employed to improve indistinguishability and entanglement come at a cost of source brightness, it is unclear whether QDs can be really used as the on-demand entangled photon source needed for long-distance quantum communication.

Below, we argue that the fidelity of the swapping operation can be instead pushed to the required values with future developments of a state of the art QD-photon source. Before explaining how to accomplish this task, we now present a novel theoretical model that not only accounts quantitatively for the experimental observations, but also explains the sources of entanglement degradation and, as a consequence, pinpoints the next steps ahead.

The success of entanglement swapping critically depends on two main parameters: the initial degree of entanglement of the photon pairs and the indistinguishability of the photons involved in the BSM. In contrast with the simple approximation presented in Ref. [42], we consider the specific role of the relevant properties of the quantum emitter. The initial degree of entanglement is known to be limited by finite FSS, spurious photons from background light or multiphoton emission, and decoherence mechanisms during the intermediate step of the cascade [11]. From a theoretical point of view, it is possible [43] to introduce all these contributions in the density matrices of the initial $\left|\Phi_{E}^{+}\right\rangle$and $\left|\Phi_{L}^{+}\right\rangle$states (that are $\rho_{X_{E}, X X_{E}}$ and $\rho_{X_{L}, X X_{L}}$ ) and project the two $X$ polarization modes onto $\left|\Psi^{-}\right\rangle$[44] to describe the density matrix resulting from the swapping operation

$$
\begin{aligned}
& \rho_{X X_{E}, X X_{L}}^{\Psi-}\left(t_{E}, t_{L}\right) \\
& \quad=\operatorname{Tr}_{X_{E}, X_{L}}\left(\frac{\Pi_{X_{E}, X_{L}}^{\Psi-}\left[\rho_{X_{E}, X X_{E}}\left(t_{E}\right) \otimes \rho_{X_{L}, X X_{L}}\left(t_{L}\right)\right] \Pi_{X_{E}, X_{L}}^{\Psi-}}{N^{\Psi^{-}}}\right),
\end{aligned}
$$

where $\Pi$ is the projection operator and $N$ is a normalization factor.

The experimental density matrix $\rho_{X X_{E}, X X_{L}}^{\Psi-}$, averaged over a large number of swapping events, is estimated integrating over the exciton recombination times $t_{E}$ and $t_{L}$ as

$$
\rho_{X X_{E}, X X_{L}}^{\Psi-}=\int_{0}^{\infty} d t_{L} \int_{0}^{\infty} d t_{E} \frac{e^{-\frac{t_{L}+t_{E}}{\tau_{X}}}}{\tau_{X}^{2}} \rho_{X X_{E}, X X_{L}}^{\Psi_{-}}\left(t_{E}, t_{L}\right) .
$$

However, a value of indistinguishability between $X_{E}$ and $X_{L}$ below unity reduces the probability $p\left(\Psi^{-} \mid \mathrm{BSM}\right)$ that a joint measurement at the two outputs of the beam splitter accurately heralds $\left|\Psi^{-}\right\rangle$. We take this effect into account by calculating $\rho_{X X_{E}, X X_{L}}^{\text {swap }}$ as a weighted sum over the possible outcomes $|i\rangle$ of the BSM,

$$
\rho_{X X_{E}, X X_{L}}^{\mathrm{swap}}=\sum_{i=\Phi^{+}, \Phi^{-}, \Psi^{+}, \Psi^{-}} p(i \mid \mathrm{BSM}) \rho_{X X_{E}, X X_{L}}^{i} .
$$

Eventually (see Sec. SIX [23,27-29] for the step-by-step procedure), we can derive an analytic expression for the fidelity to $\left|\Psi^{-}\right\rangle$, which reads

$$
\begin{aligned}
& f_{X X_{E}, X X_{L}}^{\Psi-} \\
& =\frac{1}{4}\left[1+\frac{V}{2-V} k^{2}\left(g_{H, V}^{\prime(1) 2}+2 \frac{g_{H, V}^{(1) 2}}{1+\left(\frac{S \tau_{X}}{\hbar} g_{H, V}^{(1)}\right)^{2}} \frac{1}{1+\left(\frac{S \tau_{X}}{\hbar} g_{\mathrm{deph}}^{(1)}\right)^{2}}\right)\right],
\end{aligned}
$$

where $k$ is the fraction of uncorrelated photons collected from the $X X-X$ cascade, $g_{H, V}^{(1)}=1 /\left(1+\tau_{X} / \tau_{S S}\right)$, $g_{H, V}^{(1)}=1 /\left(1+\tau_{X} / \tau_{S S}+\tau_{X} / \tau_{H V}\right), \quad$ and $\quad g_{\mathrm{deph}}^{(1)}=1 /(1+$ $\left.2 \tau_{X} / T_{2}^{*}\right)$ with $\tau_{S S}, \tau_{H V}$, and $T_{2}^{*}$, respectively, defined as the spin-scattering, cross-dephasing, and pure-dephasing characteristic times.

By measuring $g_{X, X X}^{(2)}(0), \rho_{X_{E}, X X_{F}}, S, \tau_{X}$, and $V$, and taking the value of the decoherence times from the literature $[11,32,43]$ it is possible to experimentally estimate all the quantities appearing in Eq. (1) and, therefore, predict $f_{X X_{E}, X X_{L}}^{\Psi-}$ with no fitting parameters. The model returns a swapping fidelity of 0.56 ( 0.64 in absence of background light and considering beam splitter imperfections), in excellent agreement with the experimental result. As a further proof of our theoretical model, we repeat the experiments and intentionally decrease either the degree of entanglement of our source, selecting a QD with a larger FSS of $5.9(5) \mu \mathrm{eV}$, or the indistinguishability of the photons, using an emitter with a HOM visibility of 0.51(2). The comparison between these data and the model, summarized in Fig. 3(b) and discussed in more detail in the Sec. SIX [23], shows once again good agreement between experiment and theory.

Our theoretical model also shows that the swapping fidelity depends in a sublinear fashion on the photon indistinguishability and that our current setup and QD sample cannot be used to perform a Bell test without a postselection technique such as narrow time gating (not suitable for applications, as mentioned above). However, our model can estimate the swapping fidelity for any QD photon source and, considering the best values of entanglement and indistinguishability available from the literature, we can predict whether QDs can be suitable for quantum communication. Discarding approaches using postselection we focus on the following works: (i) Huber et al. [11], who have demonstrated that QDs can deliver entangled photons with fidelities up to $98 \%$ using micromachined piezoactuators to suppress the FSS; (ii) J. Liu et al. [13], and H. Wang et al. [14], who have very recently shown that QDs embedded in circular Bragg resonators can deliver photons with indistinguishability up to $90 \%$, together with remarkably 

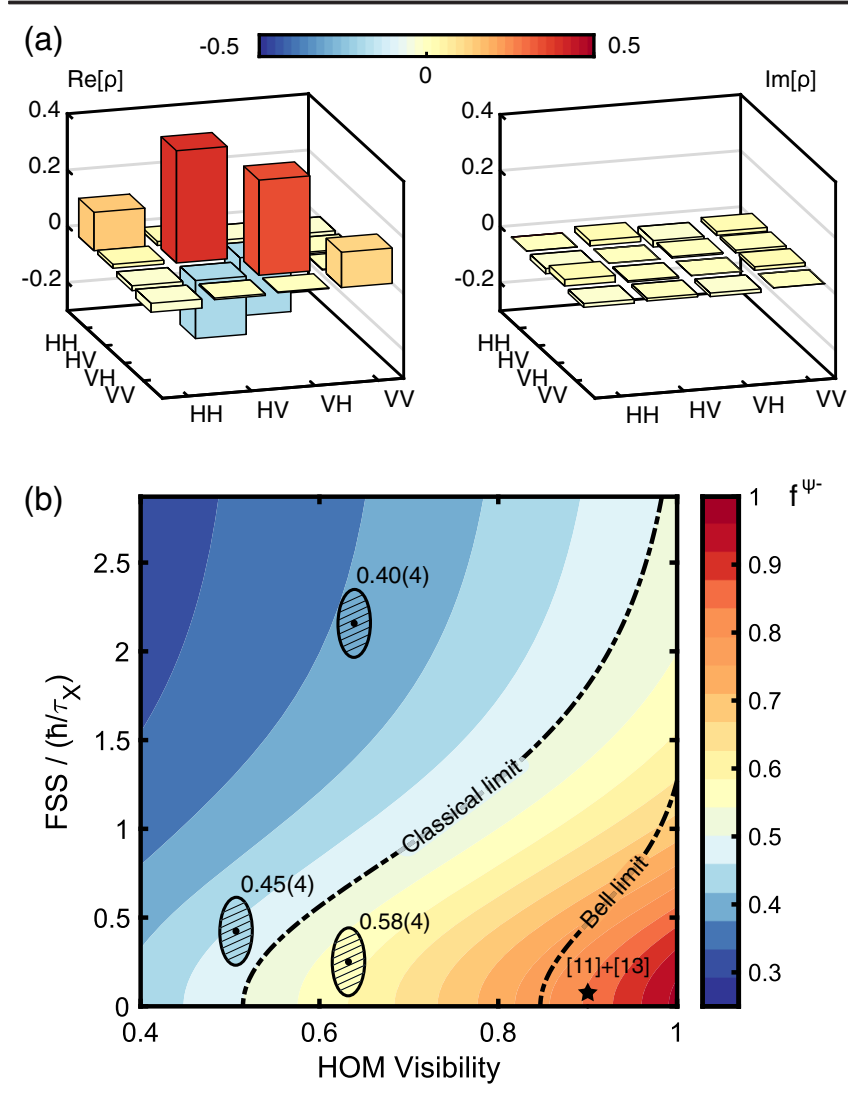

FIG. 3. (a) Real and imaginary part of the two-photon density matrix reconstructed from measurement that probes the polarization state of the $X X$ photons selected in conjunction with a BSM on their entangled partners. The matrix refers to the QD with FSS equal to $0.6 \mu \mathrm{eV}$ and HOM visibility of 0.63 . (b) Contour plot showing the expected fidelity to the $\left|\Psi^{-}\right\rangle$state as a function of HOM visibility and the ratio between FSS and radiative lifetime. The ellipses refer to the QDs considered in this work, are labeled with the experimental values of fidelity to $\left|\Psi^{-}\right\rangle$, and their semi-axes are given by the uncertainties on FSS and visibility. The star indicates the expected value of fidelity to $\left|\Psi^{-}\right\rangle$for a QD combining broadband Purcell enhancement $[13,14]$ and strain tuning of the FSS [11].

high brightness. Combining the best features from the two approaches, we can use our theoretical model to estimate the swapping fidelity and concurrence, which are found to be 0.84 and 0.67 , respectively. These values are sufficient to violate Bell's inequality [45], as shown in Fig. 3(b), and implement error-correction protocols [39]. Besides, further improvements are envisaged when looking forward to extending the protocol towards a complete BSM [46,47], in which the Bell states are additionally discerned by polarizing beam splitters and multiple detectors, and we would expect a swapping fidelity of 0.92 and a concurrence of 0.84. Our analysis implies that an "ideal" device would consist of a photonic cavity, that provides significant broadband Purcell enhancement $[48,49]$ as in (ii), integrated onto a micromachined piezoelectric actuator, as in (i). The practical implementation of such a device is certainly a grand challenge, but our theoretical and experimental work on entanglement swapping anticipates that the strive to reach this goal is certainly worth the efforts, as the realization of the ideal "on-demand entangled photon source" could be revolutionary for quantum communication science and technology.

This work was financially supported by the European Research Council (ERC) under the European Union's Horizon 2020 Research and Innovation Programme (SPQRel, Grant Agreement No. 679183), the Austrian Science Fund (FWF; P 29603), and the European Union Seventh Framework Programme (FP7/2007-2013) under Grant Agreement No. 601126 (HANAS). K. D. J. and R. T. acknowledge the COST Action MP1403, supported by COST (European Cooperation in Science and Technology). A. R. acknowledges the Linz Institute of Technology for support and Y. Huo and G. Weihs for fruitful discussions. K. D. Z. gratefully acknowledges funding by the Dr. Isolde Dietrich foundation.

Note added.-After submission we became aware of a related work [50] that uses temporal postselection techniques and a different experimental setup for the BSM.

*These authors contributed equally to this work. rinaldo.trotta@uniroma1.it

[1] J.-W. W. Pan, D. Bouwmeester, H. Weinfurter, and A. Zeilinger, Phys. Rev. Lett. 80, 3891 (1998).

[2] H. Bernien, B. Hensen, W. Pfaff, G. Koolstra, M. S. Blok, L. Robledo, T. H. Taminiau, M. Markham, D. J. Twitchen, L. Childress, and R. Hanson, Nature (London) 497, 86 (2013).

[3] W. Rosenfeld, D. Burchardt, R. Garthoff, K. Redeker, N. Ortegel, M. Rau, and H. Weinfurter, Phys. Rev. Lett. 119, 010402 (2017).

[4] A. Zeilinger, Phys. Scr. 92, 072501 (2017).

[5] S. Wehner, D. Elkouss, and R. Hanson, Science 362, eaam9288 (2018).

[6] S. K. Liao et al., Nat. Photonics 11, 509 (2017).

[7] L. Caspani, C. Xiong, B. J. Eggleton, D. Bajoni, M. Liscidini, M. Galli, R. Morandotti, and D. J. Moss, Light Sci. Appl. 6, e17100 (2017).

[8] N. Somaschi, V. Giesz, L. De Santis, J. C. Loredo, M. P. Almeida, G. Hornecker, S. L. Portalupi, T. Grange, C. Antón, J. Demory, C. Gómez, I. Sagnes, N. D. Lanzillotti-Kimura, A. Lemaítre, A. Auffeves, A. G. White, L. Lanco, and P. Senellart, Nat. Photonics 10, 340 (2016).

[9] X. Ding, Y. He, Z.-C. Duan, N. Gregersen, M.-C. Chen, S. Unsleber, S. Maier, C. Schneider, M. Kamp, S. Höfling, C.-Y. Lu, and J.-W. Pan, Phys. Rev. Lett. 116, 020401 (2016).

[10] K. D. Jöns, L. Schweickert, M. A. M. Versteegh, D. Dalacu, P. J. Poole, A. Gulinatti, A. Giudice, V. Zwiller, and M. E. Reimer, Sci. Rep. 7, 1700 (2017).

[11] D. Huber, M. Reindl, S. F. Covre da Silva, C. Schimpf, J. Martín-Sánchez, H. Huang, G. Piredda, J. Edlinger, A. Rastelli, and R. Trotta, Phys. Rev. Lett. 121, 033902 (2018). 
[12] Y. Chen, M. Zopf, R. Keil, F. Ding, and O. G. Schmidt, Nat. Commun. 9, 2994 (2018).

[13] J. Liu, R. Su, Y. Wei, B. Yao, S. F. C. da Silva, Y. Yu, J. Iles-Smith, K. Srinivasan, A. Rastelli, J. Li, and X. Wang, Nat. Nanotechnol. 14, 586 (2019).

[14] H. Wang, H. Hu, T.-H. Chung, J. Qin, X. Yang, J.-P. Li, R.-Z. Liu, H.-S. Zhong, Y.-M. He, X. Ding, Y.-H. Deng, Q. Dai, Y.-H. Huo, S. Höfling, C.-Y. Lu, and J.-W. Pan, Phys. Rev. Lett. 122, 113602 (2019).

[15] C. Varnava, R. M. Stevenson, J. Nilsson, J. SkibaSzymanska, B. Dzurňák, M. Lucamarini, R. V. Penty, I. Farrer, D. A. Ritchie, and A. J. Shields, npj Quantum Inf. 2, 16006 (2016).

[16] M. Reindl, D. Huber, C. Schimpf, S. F. C. da Silva, M. B. Rota, H. Huang, V. Zwiller, K. D. Jöns, A. Rastelli, and R. Trotta, Sci. Adv. 4, eaau1255 (2018).

[17] R. Trotta, E. Zallo, C. Ortix, P. Atkinson, J. D. Plumhof, J. van den Brink, A. Rastelli, and O. G. Schmidt, Phys. Rev. Lett. 109, 147401 (2012).

[18] M. Müller, S. Bounouar, K. D. Jöns, M. Glässl, and P. Michler, Nat. Photonics 8, 224 (2014).

[19] S. Stufler, P. Machnikowski, P. Ester, M. Bichler, V. M. Axt, T. Kuhn, and A. Zrenner, Phys. Rev. B 73, 125304 (2006).

[20] H. Jayakumar, A. Predojević, T. Huber, T. Kauten, G. S. Solomon, and G. Weihs, Phys. Rev. Lett. 110, 135505 (2013).

[21] S. L. Braunstein and A. Mann, Phys. Rev. A 51, R1727 (1995).

[22] Y. H. Huo, A. Rastelli, and O. G. Schmidt, Appl. Phys. Lett. 102, 152105 (2013).

[23] See Supplemental Material at http://link.aps.org/ supplemental/10.1103/PhysRevLett.123.160501 for the procedure of sample fabrication, which includes Ref. [24], for a more in-depth discussion of the source properties, which includes Refs. [25,26], for a step-by-step derivation of Eq. (1), which includes Refs. [27-29], and for additional information concerning the experimental setup and data analysis.

[24] C. Heyn, A. Stemmann, T. Köppen, C. Strelow, T. Kipp, M. Grave, S. Mendach, and W. Hansen, Appl. Phys. Lett. 94, 183113 (2009).

[25] R. J. Young, R. M. Stevenson, A. J. Hudson, C. A. Nicoll, D. A. Ritchie, and A. J. Shields, Phys. Rev. Lett. 102, 030406 (2009).

[26] J.-P. Jahn, M. Munsch, L. Béguin, A. V. Kuhlmann, M. Renggli, Y. Huo, F. Ding, R. Trotta, M. Reindl, O. G. Schmidt, A. Rastelli, P. Treutlein, and R. J. Warburton, Phys. Rev. B 92, 245439 (2015).

[27] N. Lütkenhaus, J. Calsamiglia, and K.-A. Suominen, Phys. Rev. A 59, 3295 (1999).

[28] T. Legero, T. Wilk, A. Kuhn, and G. Rempe, Appl. Phys. B 77, 797 (2003)

[29] B. Kambs and C. Becher, New J. Phys. 20, 115003 (2018).
[30] D. Huber, M. Reindl, Y. Huo, H. Huang, J. S. Wildmann, O. G. Schmidt, A. Rastelli, and R. Trotta, Nat. Commun. 8, 15506 (2017).

[31] F. Basso Basset, S. Bietti, M. Reindl, L. Esposito, A. Fedorov, D. Huber, A. Rastelli, E. Bonera, R. Trotta, and S. Sanguinetti, Nano Lett. 18, 505 (2018).

[32] M. Reindl, K. D. Jöns, D. Huber, C. Schimpf, Y. Huo, V. Zwiller, A. Rastelli, and R. Trotta, Nano Lett. 17, 4090 (2017).

[33] L. Schweickert, K. D. Jöns, K. D. Zeuner, S. F. Covre da Silva, H. Huang, T. Lettner, M. Reindl, J. Zichi, R. Trotta, A. Rastelli, and V. Zwiller, Appl. Phys. Lett. 112, 093106 (2018).

[34] R. M. Stevenson, J. Nilsson, A. J. Bennett, I. Farrer, D. A. Ritchie, and A. J. Shields, Nat. Commun. 4, 2859 (2013).

[35] J. B. Altepeter, E. R. Jeffrey, P. G. Kwiat, S. Tanzilli, N. Gisin, and A. Acín, Phys. Rev. Lett. 95, 033601 (2005).

[36] D. F. V. James, P. G. Kwiat, W. J. Munro, and A. G. White, Phys. Rev. A 64, 052312 (2001).

[37] A. G. White, A. Gilchrist, G. J. Pryde, J. L. O'Brien, M. J. Bremner, and N. K. Langford, J. Opt. Soc. Am. B 24, 172 (2007).

[38] W. J. Munro, K. Nemoto, and A. G. White, J. Mod. Opt. 48, 1239 (2001).

[39] J. Huwer, R. M. Stevenson, J. Skiba-Szymanska, M. B. Ward, A. J. Shields, M. Felle, I. Farrer, D. A. Ritchie, and R. V. Penty, Phys. Rev. Applied 8, 024007 (2017).

[40] N. Akopian, N. H. Lindner, E. Poem, Y. Berlatzky, J. Avron, D. Gershoni, B. D. Gerardot, and P. M. Petroff, Phys. Rev. Lett. 96, 130501 (2006).

[41] J. Iles-Smith, D. P. S. McCutcheon, A. Nazir, and J. Mørk, Nat. Photonics 11, 521 (2017).

[42] R. M. Stevenson, C. L. Salter, J. Nilsson, A. J. Bennett, M. B. Ward, I. Farrer, D. A. Ritchie, and A. J. Shields, Phys. Rev. Lett. 108, 040503 (2012).

[43] A. J. Hudson, R. M. Stevenson, A. J. Bennett, R. J. Young, C. A. Nicoll, P. Atkinson, K. Cooper, D. A. Ritchie, and A. J. Shields, Phys. Rev. Lett. 99, 266802 (2007).

[44] B. T. Kirby, S. Santra, V. S. Malinovsky, and M. Brodsky, Phys. Rev. A 94, 012336 (2016).

[45] S. Ghosh, G. Kar, A. S. De, and U. Sen, Phys. Rev. A 64, 044301 (2001).

[46] Y.-H. Kim, S. P. Kulik, and Y. Shih, Phys. Rev. Lett. 86, 1370 (2001).

[47] C. Nölleke, A. Neuzner, A. Reiserer, C. Hahn, G. Rempe, and S. Ritter, Phys. Rev. Lett. 110, 140403 (2013).

[48] T. Huber, A. Predojević, H. Zoubi, H. Jayakumar, G. S. Solomon, and G. Weihs, Opt. Express 21, 9890 (2013).

[49] F. Troiani, Phys. Rev. B 90, 245419 (2014).

[50] M. Zopf, R. Keil, Y. Chen, J. Yang, D. Chen, F. Ding, and O. G. Schmidt, following Letter, Phys. Rev. Lett. 123, 160502 (2013).. 